\title{
dbCPG: A web resource for cancer predisposition genes
}

Research Paper

\author{
Ran Wei ${ }^{1}$, Yao Yao', Wu Yang ${ }^{2}$, Chun-Hou Zheng ${ }^{2,3}$, Min Zhao ${ }^{4}$, Junfeng Xia ${ }^{1,3}$ \\ ${ }^{1}$ Institute of Health Sciences, School of Computer Science and Technology, Anhui University, Hefei, Anhui, 230601, China \\ ${ }^{2}$ College of Electrical Engineering and Automation, Anhui University, Hefei, Anhui, 230601, China \\ ${ }^{3}$ Co-Innovation Center for Information Support and Assurance Technology, Anhui University, Hefei, Anhui, 230601, China \\ ${ }^{4}$ School of Engineering, Faculty of Science, Health, Education and Engineering, University of Sunshine Coast, Maroochydore \\ DC, Queensland, 4558, Australia
}

Correspondence to: Junfeng Xia, email: jfxia@ahu.edu.cn

Keywords: cancer predisposition gene, database, functional annotation, gene prioritization, network module

Received: November 08, $2015 \quad$ Accepted: April 28, $2016 \quad$ Published: May 12, 2016

\section{ABSTRACT}

\begin{abstract}
Cancer predisposition genes (CPGs) are genes in which inherited mutations confer highly or moderately increased risks of developing cancer. Identification of these genes and understanding the biological mechanisms that underlie them is crucial for the prevention, early diagnosis, and optimized management of cancer. Over the past decades, great efforts have been made to identify CPGs through multiple strategies. However, information on these CPGs and their molecular functions is scattered. To address this issue and provide a comprehensive resource for researchers, we developed the Cancer Predisposition Gene Database (dbCPG, Database URL: http://bioinfo.ahu.edu. $\mathrm{cn}: \mathbf{8 0 8 0} / \mathrm{dbCPG} /$ index.jsp), the first literature-based gene resource for exploring human CPGs. It contains 827 human (724 protein-coding, 23 non-coding, and 80 unknown type genes), 637 rats, and 658 mouse CPGs. Furthermore, data mining was performed to gain insights into the understanding of the CPGs data, including functional annotation, gene prioritization, network analysis of prioritized genes and overlap analysis across multiple cancer types. A user-friendly web interface with multiple browse, search, and upload functions was also developed to facilitate access to the latest information on CPGs. Taken together, the dbCPG database provides a comprehensive data resource for further studies of cancer predisposition genes.
\end{abstract}

\section{INTRODUCTION}

Cancer, as the second leading cause of death, is a major public health problem in the world. For instance, it is estimated that there are $1,658,370$ new cancer cases and 589,430 cancer deaths in the United States in 2015 [1]. At least $3 \%$ of all cancers are hereditary, meaning a germline pathogenic mutation can contribute to cancer development [2]. Genes in which germline mutations increase the risks of developing cancer are called cancer predisposition genes (CPGs) [2]. It has long been acknowledged that the most of CPGs play significant role in fundamental biological processes such as DNA repair and cell cycle regulation [3]. Most CPGs act as tumor suppressors with mutations that abolish their function and contribute to the development of a cancer, only a few CPGs predisposed to cancer is the result of gain-of-function mutations [2]. Besides, the contribution of CPG mutations across cancer types is highly variable. For example, it was estimated that around $5-10 \%$ of breast cancers are due to germline mutations in CPGs such as BRCA1 and BRCA2 [4], while lung cancers are thought to be more strongly related to environment components.

The identification of CPG has a substantial impact on cancer detection and prevention [5]. As a result, many small-scale studies such as candidate gene approaches and high-throughput strategies like genome-wide mutation analyses (including exome and genome sequencing) have been applied onto the studies of CPG over the past decades. This has resulted in generation of enormous data and revelation of hundreds of disease-associated genomic markers in cancer patients, thus providing researchers important resources to potentially explore the molecular mechanisms and identify CPGs.

In the past few years, a larger number of database have emerged which mainly focused on a particular class 
of cancer genes as exemplified by tumor suppressor gene database [6], candidate cancer gene database [7], and cancer-related immunological gene database [8]. However, to the best of our knowledge, there is no database that focuses on CPGs. To fill this gap, we developed a comprehensive literature based database called dbCPG (Cancer Predisposition Gene Database). Aiming to efficiently integrate and analyze all or most of the published CPGs, we firstly performed a collection and review of peer-reviewed literature from databases such as Rahman's data [2], PubMed abstract (http:/www.ncbi.nlm.nih.gov/ pubmed), GeneReview [9], Online Mendelian Inheritance in Man (OMIM) [10] and Gene Reference Into Function (GeneRIF) [11]. Then we manually checked and obtained a total of 827 human (724 protein-coding, 23 non-coding and 80 unknown type genes (the type of gene is labelled as 'unknown type' in NCBI)), 637 rat and 658 mouse CPGs. To provide a comprehensive data source for cancer predisposition genes, the $\mathrm{dbCPG}$ integrates multitudinous annotation information for each $\mathrm{CPG}$, including general information from NCBI, gene expressions from Expression Atlas [12], methylation sites from DiseaseMeth database [13], post-translational modification (PTM) information from dbPTM [14], germline mutation data from ClinVar [15], interacting partners from PINA [16], pathway information from MSigDB [17], and drug information from DGIdb [18]. As the first database for CPGs, dbCPG provides not only a comprehensive resource of CPGs for the cancer research community but also provides useful information for clinical application, such as diagnosis, optimized management and prevention of cancer.

\section{RESULTS AND DISCUSSION}

\section{Representative entry in dbCPG}

The gene entries in $\mathrm{dbCPG}$ can be easily accessed in a variety of ways. The main page for each gene displays 8 annotation categories, including 'General Information', 'Expression', 'Methylation', 'PTM', 'Mutation', 'Interaction', 'Pathway' and 'Drug' category (Figure 1).

In the 'General Information' category, basic gene information, nucleotide sequence and protein sequence are displayed in a tabular view. Summaries of literature evidence and data sources are also provided. The 'Expression' category provides a tabular data which exhibits the $P$-value, $\log 2$ fold change and T-statistic with different comparisons, like 'high grade serous ovarian carcinoma' vs 'normal', which were collected from Expression Atlas [12] with the keywords 'cancer' AND 'Homo sapiens'. In the 'Methylation' category, we used the textual interfaces to depict the methylation information, which were obtained from DiseaseMeth database [13]. The chromosome, methylation location and their respective sources are displayed. In the 'PTM' category, we displayed the PTM type with different amino acid modified location.
We also showed the PubMed ID and data sources. All of these data were obtained from dbPTM [14]. The 'Mutation' category presents different types of germline mutations that were extracted from ClinVar [15]. In the 'Interaction' category, users can view the interaction partners and types, which were derived from PINA database [16]. For each CPG, we offered its involved pathways in the 'Pathway' category, which were obtained from MSigDB [17]. In the 'Drug' category, users can explore drug related information such as drug name, interaction type and data source, which were extracted from DGIdb database [18].

\section{Database statistics}

The current version of dbCPG contains 827 human, 637 rat and 658 mouse CPGs. Descriptions on the data sources and functional information are summarized in Table 1. For humans, each chromosome carries at least one CPG (Figure 2A), and most of CPGs is located on chromosome 1 (75 CPGs) and 11 (66 CPGs). In our database, the human CPGs were retrieved from five data sources (Figure 2B). Supplementary Figure S1 is a Venn diagram illustrating the overlapping CPGs among these five data sources. Since most of GeneRIFs were extracted from the title or abstract of the corresponding scientific paper [19], and OMIM is an authoritative catalog of human genes and traits, it is not surprising to see that a large proportion of human CPGs in dbCPG were obtained from GeneRIF (56.71\%) or OMIM (43.77\%).

Among the 827 human CPGs, the majority of them (724) are protein-coding genes (Figure 2C). Only 23 belong to non-coding genes. In addition, $80 \mathrm{CPGs}$ are labelled as 'unknown type' in dbCPG based on the annotation in NCBI. As CPGs have been reported in different types of cancer, this information were also included in dbCPG. In total, there are 90 types of cancer with reported CPGs in our database, of which the top 17 major cancer types contain at least $21 \mathrm{CPGs}$ (Figure 2D). And breast cancer is the most frequent tumor with the number of CPGs.

We also investigated the overlap between human CPGs and the known cancer genes with somatic mutations (Figure 3). 570 somatically mutated cancer genes were obtained from the COSMIC (Catalogue of Somatic Mutations in Cancer) database [20]. Of these, 218 are also known to be CPGs. These data reveal that $38 \%$ of somatically mutated cancer genes can cause predisposition to cancer when they have germline mutations. Conversely, we also see that about $26 \%$ of CPGs can contribute to carcinogenesis when they have somatic mutations. Considering the fact that cancer is a genetic disease driven by a combination of germline mutations coupled with the acquisition of somatic mutations, the integrated analysis of germline and somatic data can facilitate identification of likely pathogenic mutations and new cancer genes that are not readily identified by studying each data in isolation $[21,22]$. 


\section{Biological features of protein-coding genes in dbCPG}

As the majority of CPGs in our database are protein-coding genes (724 human protein-coding CPGs in Supplementary Table S1), we performed the pathway enrichment and disease association analyses on the 724 protein coding genes to better understand the biological function using the online tool KOBAS [23]. We collected the pathways and disease with an adjusted $P$-value $<0.05$ as calculated by hypergeometric test followed by the Benjamini-

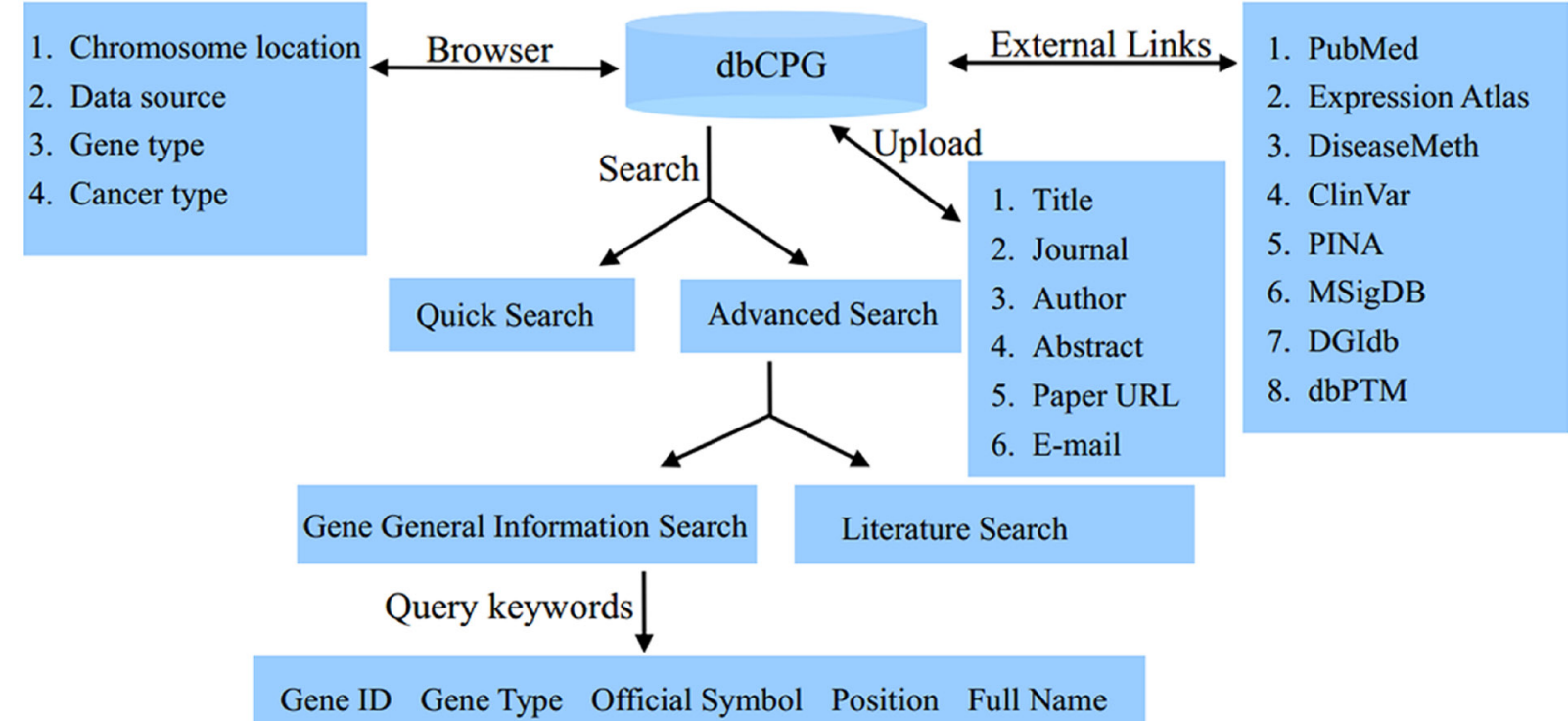

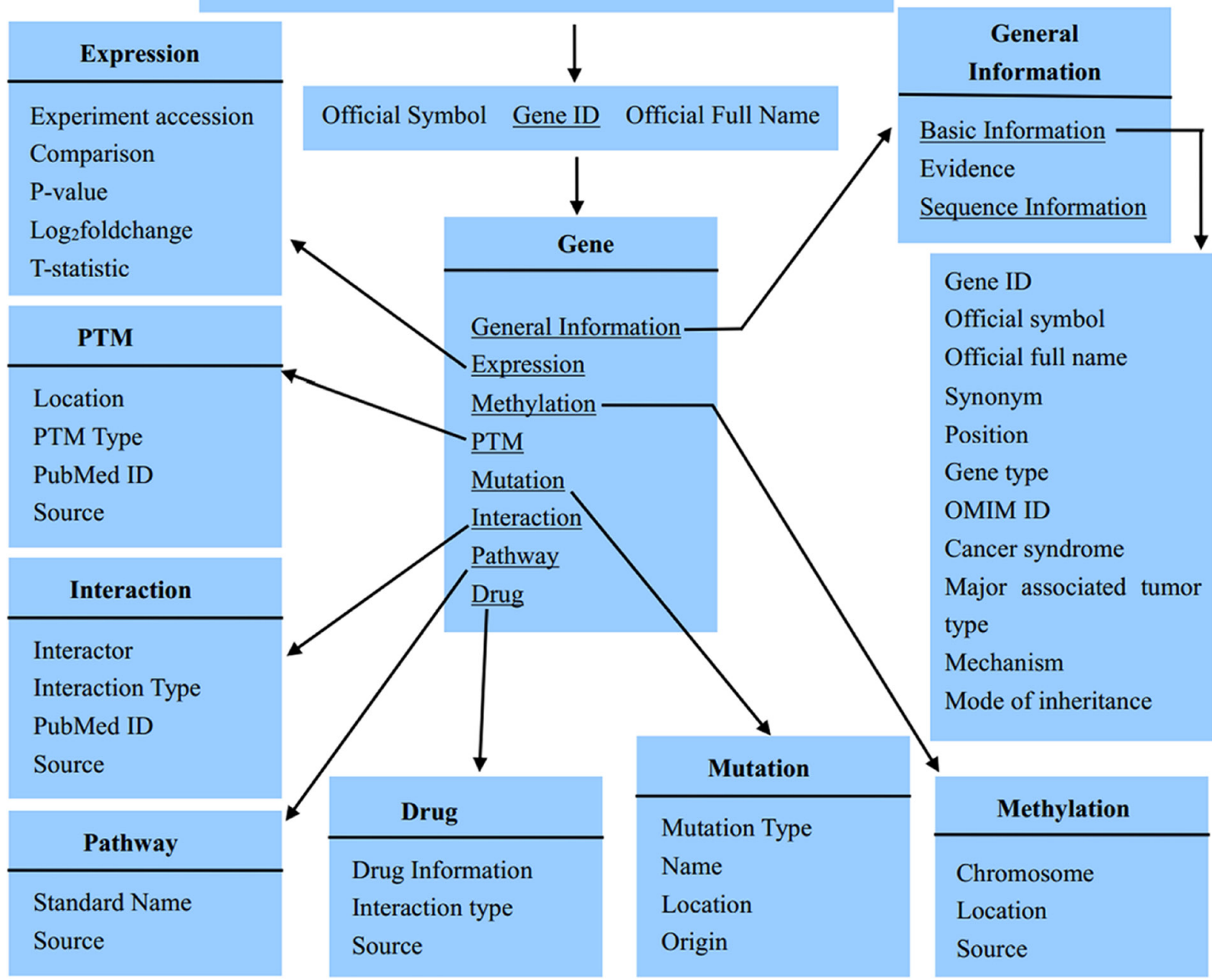

Figure 1: The database structure of dbCPG. 
Table 1: Annotation entry statistics for 827 CPGs

\begin{tabular}{|l|c|c|l|}
\hline \multicolumn{1}{c|}{ Data category } & Related entries & \multicolumn{1}{c}{ Annotated CPGs } & \multicolumn{1}{c|}{ Content/sources } \\
\hline Human CPGs & 827 & 827 & $\begin{array}{l}\text { Gene ID, official symbol, official full name, synonym, } \\
\text { position, gene type, OMIM ID from Entrez gene } \\
\text { database; cancer syndrome, major associated tumor } \\
\text { type, mechanism of action of CPG mutations, mode of } \\
\text { inheritance from PubMed }\end{array}$ \\
\hline Rat CPGs & 637 & 637 & Rat CPGs mapped from HomoloGene \\
\hline Mouse CPGs & 658 & 658 & $\begin{array}{l}\text { Mouse CPGs mapped from MGI Human Mouse } \\
\text { Orthologs }\end{array}$ \\
\hline Literature & 2097 & 805 & Literature evidence for CPGs \\
\hline OMIM & 22 & 22 & Disorder description for CPGs \\
\hline Expression & 8873 & 654 & Expression Atlas database \\
\hline Methylation & 5292 & 695 & DiseaseMeth database \\
\hline PTM & 11701 & 366 & dbPTM \\
\hline Germline mutation & 29816 & 420 & ClinVar \\
\hline Interaction & 20004 & 610 & PINA database \\
\hline Pathway & 8640 & 580 & MsigDB database \\
\hline Drug & 1651 & 133 & DGIdb database \\
\hline
\end{tabular}

*CPG is short for cancer predisposition gene, MGI is short for mouse genome informatics, PTM is short for post-translational modification.

Hochberg correction. As shown in Supplementary Table S3, a lot of the enriched pathways, such as 'pathways in cancer', 'constitutive PI3K/AKT signaling in cancer', 'p53 signaling pathway', and 'PI3K/AKT signaling in cancer' are cancer relevant. Among the top 20 enriched disease (Supplementary Table S4), there are 18 various types of cancer on the enriched list, including breast cancer, colorectal cancer, prostate cancer, lung cancer and ovarian cancer (Table 2).
To obtain comprehensive biological features, we also conducted enrichment tests on 724 human protein-coding CPGs by using DAVID server [24]. Statistically significant gene ontology (GO) terms and over-represented InterPro domain [25] were selected by an adjusted $P$-value $<0.05$ calculated by hypergeometric test followed by the BenjaminiHochberg correction. Using the complete human genes as background, the 724 protein-coding CPGs were over-

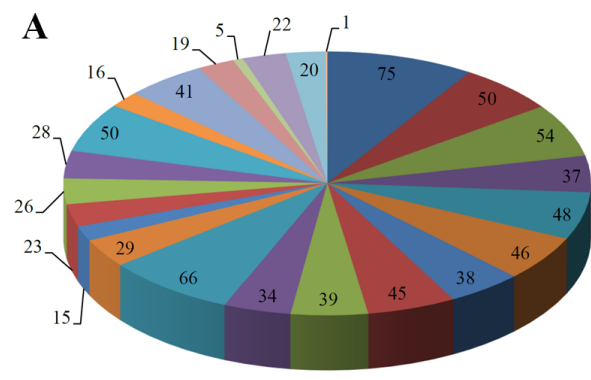

C

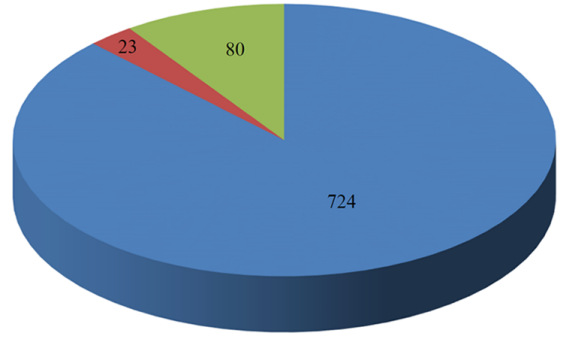

m $\mathrm{Chr} 1$ - $\mathrm{Chr} 2$

- $\mathrm{Chr} 3=\mathrm{Chr} 4$

m $\mathrm{Chr} 5$ = Chr 6

$=\mathrm{Chr} 7 \mathrm{Chr} 8$

$=\mathrm{Chr} 9=\mathrm{Chr} 10$

=Chr $11=\mathrm{Chr} 12$

$=\mathrm{Chr} 13=\mathrm{Chr} 14$

$=\mathrm{Chr} 15=\mathrm{Chr} 16$

m $\mathrm{Chr} 17$ - $\mathrm{Chr} 18$

$=\mathrm{Chr} 19=\mathrm{Chr} 20$

$\varpi \mathrm{Chr} 21 \quad \mathrm{~m} \mathrm{Chr} 22$ $₫ \mathrm{Chr} \mathrm{X} \equiv \mathrm{Chr} \mathrm{Y}$

- Protein coding

- Non-coding

= Unknown type
B

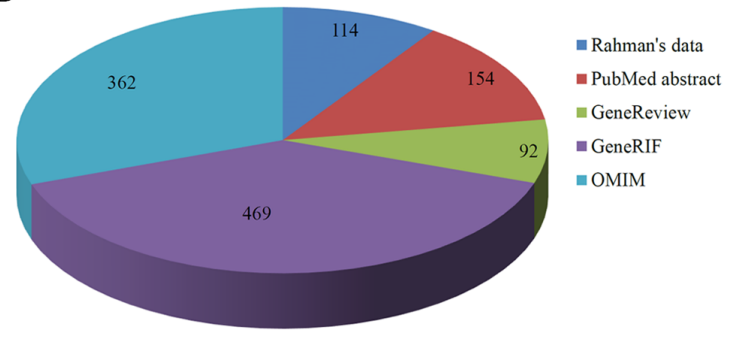

D

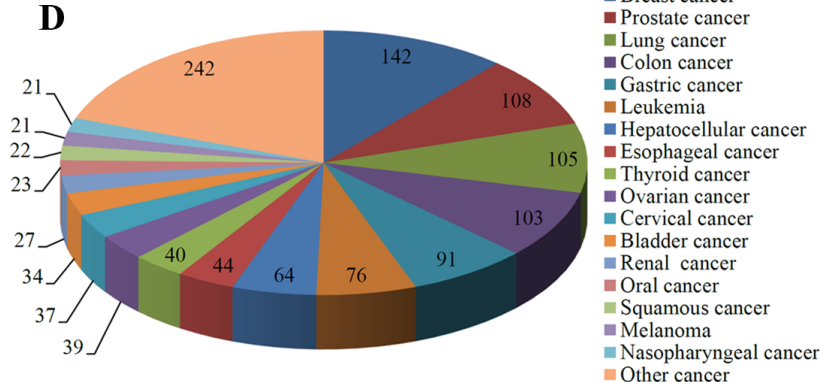

Figure 2: Data statistics based on (A) Chromosome location, (B) Data source, (C) Gene type, and (D) Cancer type in human CPGs. 
represented in regulation of biological process, regulation of cellular process, regulation of cell proliferation and cell death according to GO Biological Processes terms (Supplementary Table S5). As shown in Supplementary Table S6, the most commonly represented InterPro domains were mainly related to kinase activities such as 'tyrosine protein kinase, active site', 'tyrosine protein kinase', 'protein kinase, ATP binding site' and 'Protein kinase, core', which highlight important roles of kinase activity in CPGs.

\section{The common CPGs across multiple cancer types}

Based on the literature review, we provided all the CPGs in dbCPG with cancer type information. We grouped all the CPGs into 90 cancer types. The number of CPGs detected per cancer type varies considerably (range 1-144), with four types having more than 100 CPGs (cancers of breast, prostate, lung, and colon) and 31 types having only $1 \mathrm{CPG}$. To investigate the common mechanism of CPGs in different cancer types, we focused on the top 17 cancer types associated with more than 20 genes (Supplementary Table S7). Based on the common genes in the 17 cancer types, the overlapping relationships were plotted in Figure 4. The plot includes three outer rings, which represent relative contribution of other cancer types to the cancer types totals. It revealed that the multiple cancer types shared potential predisposition mechanisms. For example, we found 221 CPGs shared in two or more cancer types (Supplementary Table S8). Strikingly, there are three common CPGs (GSTM1, MSH6, and TP53) involved in at least 10 cancer types, in which germline mutations of these genes have been reported to increase individual susceptibility to a variety of human cancers [26-28].

\section{Prioritization of protein-coding CPGs and its enriched dense network module}

Although the 724 protein-coding genes in $\mathrm{dbCPG}$ have literature evidence based on different data sources, we didn't investigate the importance of each CPG systematically. Hence, we performed gene prioritization analysis using ToppGene web server [29]. To prioritize genes, ToppGene uses a fuzzy-based similarity measure to calculate the similarity between two types of gene set (training set and test set) based on functional annotations. In this study, we compiled a training gene lists that included 57 well-established CPGs (Supplementary Table S9), which have at least 10 literature evidences. The remaining CPGs in dbCPG were used as the test set. Based on the gene ranking results of ToppGene (Supplementary Table S10), the top ranked genes tend to have multiple evidences. Besides 57 well-studied CPGs in the training set, CTNNB1 was top ranked CPG in remaining 667 CPGs from the test set. Furthermore, functional analyses on the 100 CPGs (57 training genes and top 43 test genes) show similar distribution with the total 724 protein-coding CPGs (Supplementary Table S11-S14).

We further explored the dense modules enriched with the 100 CPGs (57 training genes and top 43 test genes) through their protein-protein interactions by using Klein-Ravi algorithm in GeneRev [30]. We identified one module which contained 107 genes (Figure 5). Of the 107 nodes, 97 of them are from our dbCPG. The remaining 10 are the novel genes that may potentially bridge the cancer predisposition gene to fully implement their cellular function. In conclusion, the majority of the $100 \mathrm{CPGs}$ connect each other and form a dense network, which also support the accuracy of our data curation.

\section{CONCLUSIONS}

This study presents a unique resource, dbCPG, for the systematic annotation of susceptibility genes in cancer predisposition. Our aim is to collect a complete and up-to-date CPG resource and make it freely accessible to users. For each CPG in our database, we provided a wide range of information, including gene expression, methylation, PTM, germline mutation, protein-protein interaction, pathway, and drug information. We will

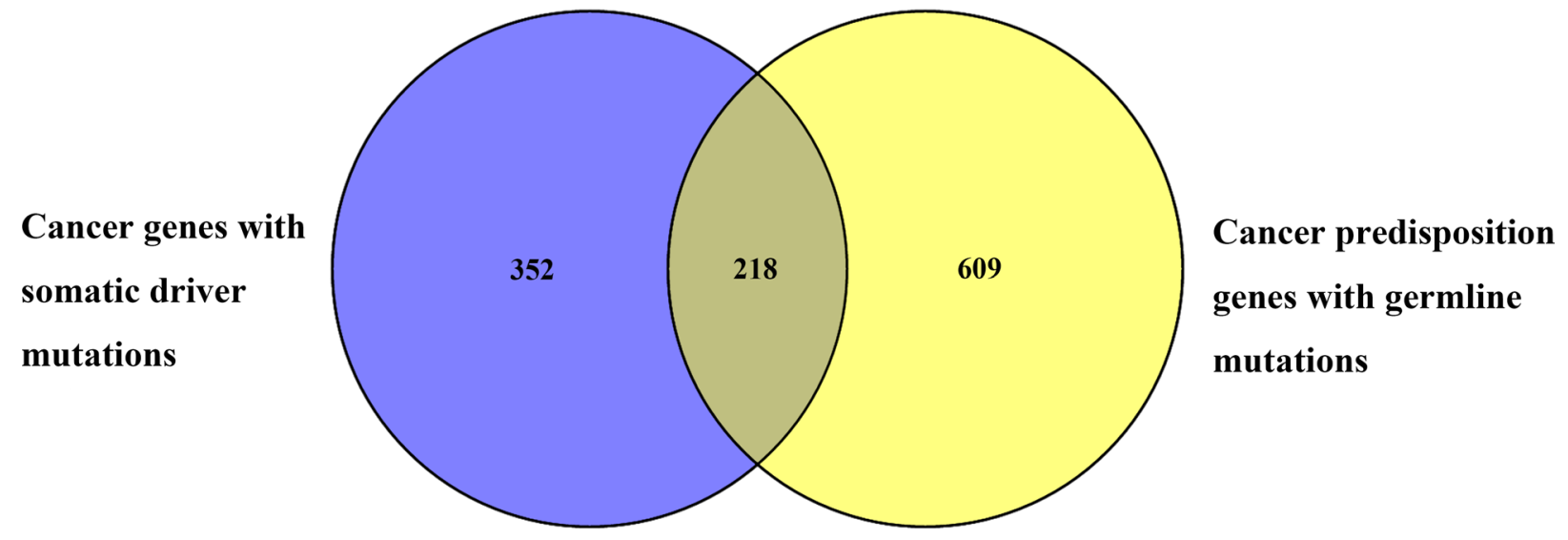

Figure 3: Overlap between cancer genes with somatic mutations and CPGs. 570 cancer genes with somatic mutations are from COSMIC of which 218 are also included within the 827 human CPGs in dbCPG. 


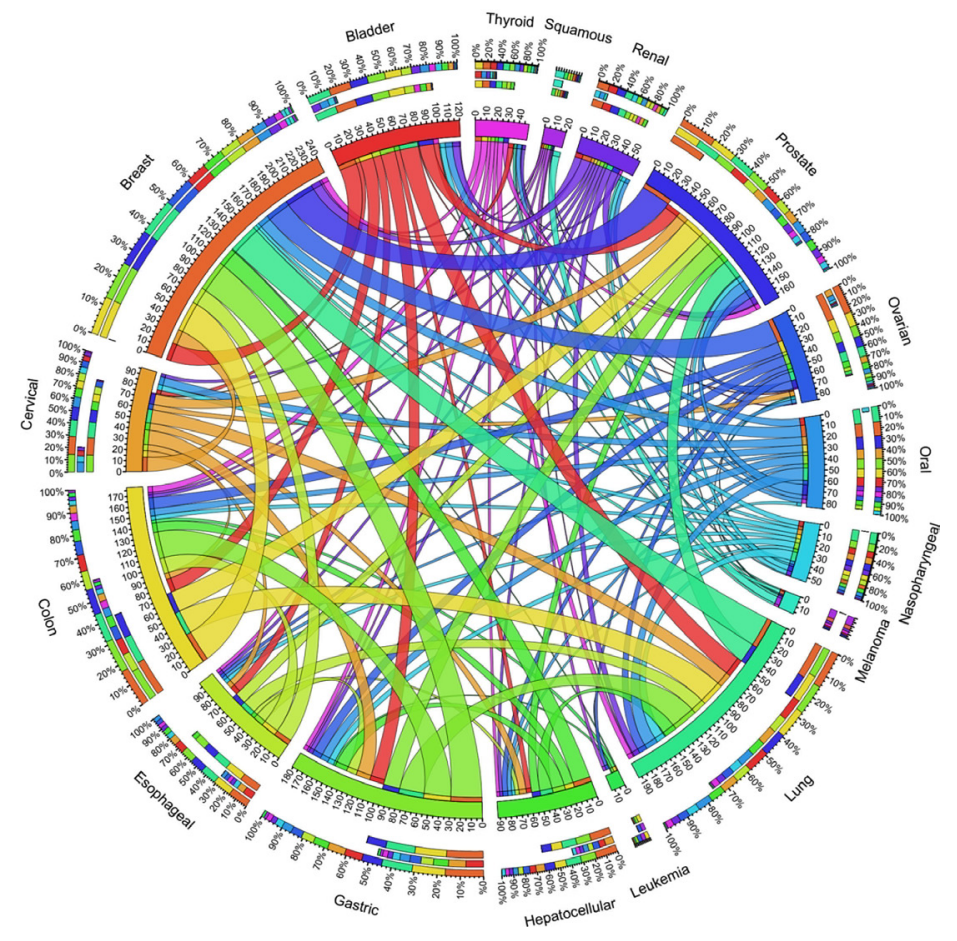

Figure 4: The shared CPGs across 17 cancer types. The length of circularly arranged segment is proportional to the total CPGs in each cancer type. The ribbons connecting different segments represent the number of shared CPGs between cancer types. The three outer rings are stacked bar plots that represent relative contribution of other cancer types to the cancer type's totals, where the innermost, middle, and outermost ring represents the number of CPGs that other cancers share with a specific cancer, the number of CPGs that a specific cancer share with the other cancers, and the sum number of CPGs among different cancer types, respectively.

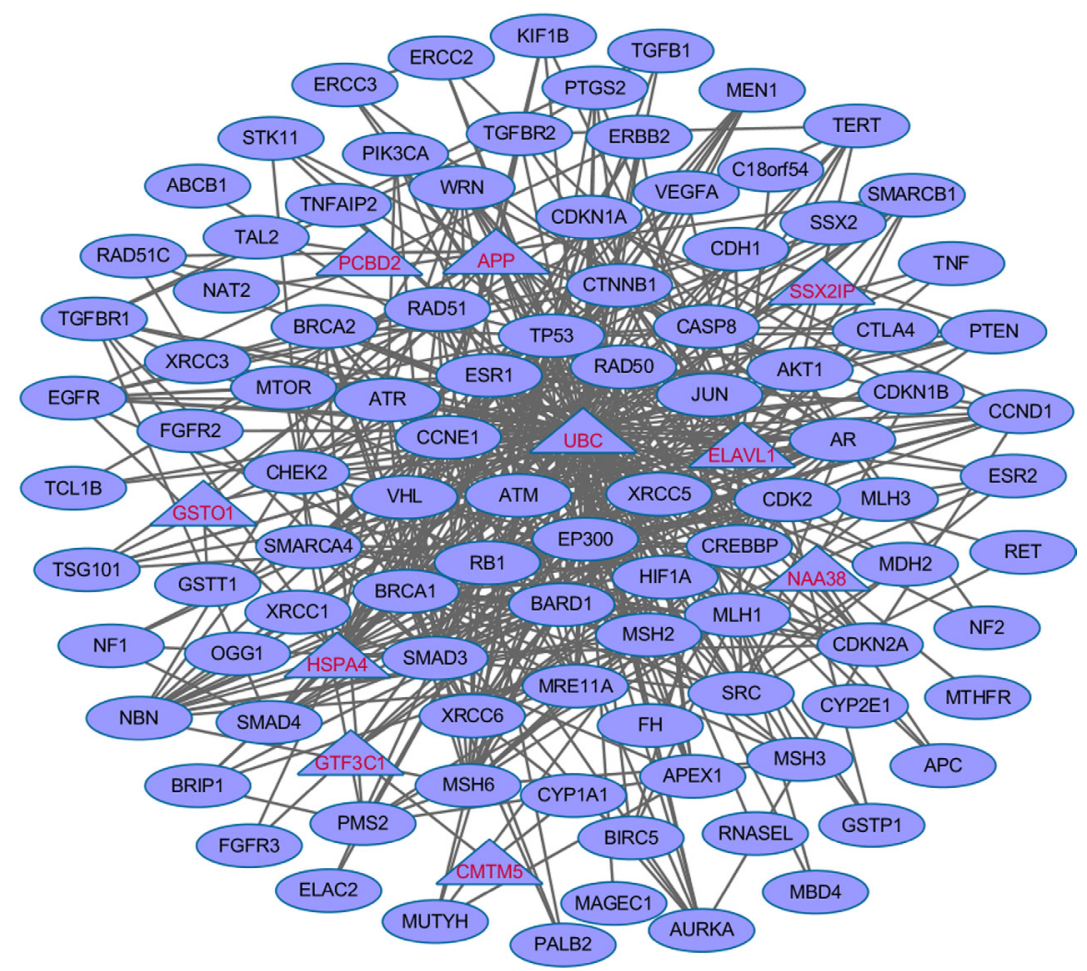

Figure 5: The enriched dense network module using the 100 CPG (57 training genes and top 43 test genes) based on protein-protein interaction data. The 97 genes in diamond are terminal genes from the 100 CPGs. The remaining 10 genes in triangle are linker genes bridged the 92 genes. 
Table 2: Top 20 enriched diseases of the 724 protein-coding CPGs

\begin{tabular}{|l|c|c|}
\hline \multicolumn{1}{|c|}{ Disease name } & Raw $\boldsymbol{P}$-value & Benjamini-Hochberg adjusted $\boldsymbol{P}$-value \\
\hline Cancer & $4.98 \mathrm{E}-30$ & $5.67 \mathrm{E}-26$ \\
\hline Breast cancer & $3.77 \mathrm{E}-29$ & $2.15 \mathrm{E}-25$ \\
\hline Colorectal cancer & $1.35 \mathrm{E}-27$ & $5.13 \mathrm{E}-24$ \\
\hline Lung cancer & $5.18 \mathrm{E}-23$ & $1.47 \mathrm{E}-19$ \\
\hline Prostate cancer & $4.04 \mathrm{E}-20$ & $9.19 \mathrm{E}-17$ \\
\hline Stomach cancer & $3.54 \mathrm{E}-17$ & $6.70 \mathrm{E}-14$ \\
\hline Bladder cancer & $8.49 \mathrm{E}-13$ & $8.05 \mathrm{E}-10$ \\
\hline Esophageal cancer & $4.68 \mathrm{E}-12$ & $2.32 \mathrm{E}-09$ \\
\hline Ovarian cancer & $2.72 \mathrm{E}-09$ & $4.76 \mathrm{E}-07$ \\
\hline Endometrial cancer & $2.77 \mathrm{E}-08$ & $2.76 \mathrm{E}-06$ \\
\hline Endometriosis & $3.59 \mathrm{E}-08$ & $3.44 \mathrm{E}-06$ \\
\hline Head and neck cancer & $3.84 \mathrm{E}-08$ & $3.55 \mathrm{E}-06$ \\
\hline Oral cancer & $1.11 \mathrm{E}-07$ & $8.32 \mathrm{E}-06$ \\
\hline Diabetes, type 1 & $1.24 \mathrm{E}-07$ & $8.94 \mathrm{E}-06$ \\
\hline Melanoma & $1.28 \mathrm{E}-07$ & $9.08 \mathrm{E}-06$ \\
\hline Stomach neoplasms & $4.64 \mathrm{E}-07$ & $2.59 \mathrm{E}-05$ \\
\hline Sarcoidosis & $6.33 \mathrm{E}-07$ & $3.32 \mathrm{E}-05$ \\
\hline Infection & $9.24 \mathrm{E}-07$ & $4.45 \mathrm{E}-05$ \\
\hline Neoplasms & $9.81 \mathrm{E}-07$ & $4.62 \mathrm{E}-05$ \\
\hline Leukemia & $1.09 \mathrm{E}-06$ & $5.02 \mathrm{E}-05$ \\
\hline
\end{tabular}

update this database on a regular basis by adding new data from literature as well as other valuable resources. It is anticipated that $\mathrm{dbCPG}$ would serve as a valuable resource to the cancer research community.

\section{MATERIALS AND METHODS}

\section{Data collection and literature curation}

The goal of dbCPG database is to provide a comprehensive resource for investigation of CPGs and their molecular mechanisms in cancer, which can freely assist cancer research community to design the experiment, understand tumorigenic mechanisms and develop useful information for clinical application. Thus, we firstly collected 114 CPGs from Rahman's Nature paper [2], where the CPGs were identified based on literature review and database evaluations. Secondly, we performed a comprehensive literature search of PubMed on 8 April 2015 using the query expression: ('cancer' [Title/Abstract] OR 'tumor' [Title/Abstract]) AND ('predisposition' [Title/Abstract] OR 'susceptibility' [Title/Abstract]) AND ('gene' [Title/Abstract] OR 'syndrome' [Title/Abstract]), with the purpose of obtaining a precise and detailed list of publications for CPGs. As a result, we obtained 1319 PubMed abstracts.
Then we extracted CPGs related sentences from the abstracts of these articles manually. We also read the full text to find the key sentences if necessary. Overall, 154 CPGs were collected from 624 related PubMed abstracts. Thirdly, 92 CPGs were identified from GeneReview [9], which is an online database mainly focused on specific heritable disease, on 20 May 2015 using the search terms: ('neoplasms' [All Fields] OR 'neoplasms' [All Fields] OR 'cancer' [All Fields]) AND ('disease susceptibility' [All Fields] OR ('disease' [All Fields] AND 'susceptibility' [All Fields]) OR 'disease susceptibility' [All Fields] OR 'predisposition' [All Fields]) AND ('genes' [All Fields] OR 'genes' [All Fields] OR 'gene' [All Fields]). Fourthly, we extracted 469 CPGs with the words: ('cancer' or 'tumor' or 'carinoma' or 'neoplasm') and ('susceptibility' or 'predisposition') from GeneRIF [11] on 23 May 2015, which is a clustering of short statements about gene function. Finally, we searched 362 CPGs derived from OMIM [10], a comprehensive database of human gene and genetic disorders, on 28 May 2015. After combing the gene sets obtained from these five data sources, we consolidated 827 human CPGs and retrieved their orthologs in rat and mouse using orthology data downloaded from HomoloGene (http://www.ncbi.nlm.nih. gov/homologene) and Mouse Genome Informatics (MGI) (http://www.informatics.jax.org). 


\section{Data mining of human CPGs}

To better understand the function of these CPGs in our dbCPG database, we retrieved comprehensive functional information from different public resources (Table 1). The basic gene information is included, such as gene ID, official symbol, official full name, synonym, position, gene type and OMIM ID from Entrez gene database and cancer syndrome, major associated type, mechanism of action of CPG mutations, and mode of inheritance from PubMed abstracts. Literature evidences were also provided. In addition, we provided functional information, including gene expression, methylation, post transcriptional modification, germline mutation, protein-protein interaction, pathway, and drug information (Figure 1). Details of these databases can be found through the cited references as well as from dbCPG.

To assess the function of 724 protein-coding CPGs, we explored the functional enrichment analysis by using two online tools, KOBAS [23] and DAVID [24]. KOBAS was used to analyze the pathway and disease, while DAVID was used to identify enriched biological themes (GO terms) and protein functional domains (InterPro terms) [25]. Then, we obtained those enriched functional terms with adjusted $P$-value less than 0.05 . Furthermore, to investigate the importance of each protein coding CPG, we performed gene prioritization using ToppGene [29]. According to the number of literature evidences, 724 protein-coding CPGs were divided into two categories, 57 genes with at least 10 literature evidences acting as training gene set, the remaining 667 genes as test gene set, and finally, Klein-Ravi algorithm in GeneRev [30] was used to search the enriched dense modules.

\section{Database construction}

We stored all the dbCPGs, annotations and related data by using MySQL (version 5.1.73), which is a popular open source and freely available database. A user-friendly web interface for browsing and searching was created using Java Server Pages technology. The database structure was shown in Figure 1, which is a systematical and detailed presentation of dbCPG.

$\mathrm{dbCPG}$ supports text query. In the home page, users can find a quick search box on the left to search by gene official symbol and gene ID. An advanced search option in search page is provided to search CPG related information, including the gene ID, gene official symbol, full name, gene type and genomic location. In addition, a search interface to access CPGs related literature provided a window for users to find more comprehensive CPG descriptions from original literature sources.

In Browser page, users can browse CPGs using genomic location, data source, gene type, species or cancer type (Figure 1). Using the chromosome browser, users can obtain a summary of the CPG lists. Clicking on the hyperlinks of the gene ID, users can access corresponding gene evidence and annotation pages. In each browser page, users can click on the hyperlinks of the specific data source, gene type, species or cancer type to obtain the corresponding CPG lists.

Aside from data retrieval from dbCPG, users are encouraged to upload additional publication information to the websites. Users may first search the dbCPG database to check if their publication has already been deposited into the database. If not, users may upload the related publication information, which will be stored in dbCPG. The new record will be forwarded to the dbCPG developer via email and will become available after a manual check and confirmation.

\section{ACKNOWLEDGMENTS AND FUNDING}

This work was supported by grants from the National Natural Science Foundation of China (31301101, 61272339 and 61272333), the Anhui Provincial Natural Science Foundation (1408085QF106), the Specialized Research Fund for the Doctoral Program of Higher Education (20133401120011), the Technology Foundation for Selected Overseas Chinese Scholars from Department of Human Resources and Social Security of Anhui Province (No. [2014]-243), the Co-Innovation Center for Information Supply and Assurance Technology of Anhui University (Y01002337) and the research start-up fellowship of University of Sunshine Coast to MZ.

\section{CONFLICTS OF INTEREST}

The authors declare that they have no competing interests.

\section{REFERENCES}

1. Siegel RL, Miller KD, Jemal A. Cancer statistics, 2015. CA Cancer J Clin. 2015; 65:5-29.

2. Rahman N. Realizing the promise of cancer predisposition genes. Nature. 2014; 505:302-308.

3. Tutt A, Ashworth A. The relationship between the roles of BRCA genes in DNA repair and cancer predisposition. Trends Mol Med. 2002; 8:571-576.

4. Economopoulou P, Dimitriadis G, Psyrri A. Beyond BRCA: new hereditary breast cancer susceptibility genes. Cancer Treat Rev. 2015; 41:1-8.

5. Byrski T, Dent R, Blecharz P, Foszczynska-Kloda M, Gronwald J, Huzarski T, Cybulski C, Marczyk E, Chrzan R, Eisen A. Results of a phase II open-label, non-randomized trial of cisplatin chemotherapy in patients with BRCA1-positive metastatic breast cancer. Breast Cancer Res. 2012; 14:R110.

6. Zhao M, Sun J, Zhao Z. TSGene: a web resource for tumor suppressor genes. Nucleic Acids Res. 2013; 41:D970-D976.

7. Abbott KL, Nyre ET, Abrahante J, Ho Y-Y, Vogel RI, Starr TK. The Candidate Cancer Gene Database: a database 
of cancer driver genes from forward genetic screens in mice. Nucleic Acids Res. 2015; 43:D844-D848.

8. Nakamura $Y$, Komiyama $T$, Furue M, Gojobori $T$, Akiyama Y. CIG-DB: the database for human or mouse immunoglobulin and $\mathrm{T}$ cell receptor genes available for cancer studies. BMC bioinformatics. 2010; 11:398.

9. Pagon RA, Adam MP, Ardinger HH, Bird TD, Dolan CR, Fong CT, Smith RJH, Stephens K. GeneReviews ${ }^{\circledR}$. Seattle (WA): University of Washington, Seattle; 1993-2015.

10. Hamosh A, Scott AF, Amberger J, Valle D, McKusick VA. Online Mendelian inheritance in man (OMIM). Hum Mutat. 2000; 15:57-61.

11. $\mathrm{Lu} \mathrm{Z}$, Cohen KB, Hunter L. GeneRIF quality assurance as summary revision. Pac Symp Biocomput. 2007; pp. 269-280.

12. Petryszak R, Burdett T, Fiorelli B, Fonseca NA, GonzalezPorta M, Hastings E, Huber W, Jupp S, Keays M, Kryvych N. Expression Atlas update - a database of gene and transcript expression from microarray-and sequencingbased functional genomics experiments. Nucleic Acids Res. 2014; 42:D926-D932.

13. Lv J, Liu H, Su J, Wu X, Liu H, Li B, Xiao X, Wang F, Wu Q, Zhang Y. DiseaseMeth: a human disease methylation database. Nucleic Acids Res. 2011:gkr1169.

14. Lee TY, Huang HD, Hung JH, Huang HY, Yang YS, Wang TH. dbPTM: an information repository of protein post-translational modification. Nucleic Acids Res. 2006; 34:D622-D627.

15. Landrum MJ, Lee JM, Riley GR, Jang W, Rubinstein WS, Church DM, Maglott DR. ClinVar: public archive of relationships among sequence variation and human phenotype. Nucleic Acids Res. 2014; 42:D980-D985.

16. Cowley MJ, Pinese M, Kassahn KS, Waddell N, Pearson JV, Grimmond SM, Biankin AV, Hautaniemi S, Wu J. PINA v2.0: mining interactome modules. Nucleic Acids Res. 2012; 40:D862-865.

17. Liberzon A, Subramanian A, Pinchback R, Thorvaldsdóttir H, Tamayo P, Mesirov JP. Molecular signatures database (MSigDB) 3.0. Bioinformatics. 2011; 27:1739-1740.

18. Griffith M, Griffith OL, Coffman AC, Weible JV, McMichael JF, Spies NC, Koval J, Das I, Callaway MB, Eldred JM. DGIdb: mining the druggable genome. Nat Methods. 2013; 10:1209-1210.

19. Gobeill J, Tbahriti I, Ehrler F, Mottaz A, Veuthey AL, Ruch P. Gene Ontology density estimation and discourse analysis for automatic GeneRIF extraction. BMC bioinformatics. 2008; 9:1-10.
20. Forbes SA, Bindal N, Bamford S, Cole C, Kok CY, Beare D, Jia M, Shepherd R, Leung K, Menzies A. COSMIC: mining complete cancer genomes in the Catalogue of Somatic Mutations in Cancer. Nucleic Acids Res. 2010:gkq929.

21. LaFramboise T, Dewal N, Wilkins K, Pe'er I, Freedman ML. Allelic selection of amplicons in glioblastoma revealed by combining somatic and germline analysis. PLoS Genet. 2010; 6:e1001086.

22. Kanchi KL, Johnson KJ, Lu C, McLellan MD, Leiserson MD, Wendl MC, Zhang Q, Koboldt DC, Xie M, Kandoth C. Integrated analysis of germline and somatic variants in ovarian cancer. Nat Commun. 2014; 5:3156-3156.

23. Xie C, Mao X, Huang J, Ding Y, Wu J, Dong S, Kong L, Gao G, Li C-Y, Wei L. KOBAS 2.0: a web server for annotation and identification of enriched pathways and diseases. Nucleic Acids Res. 2011; 39:W316-W322.

24. Huang DW, Sherman BT, Tan Q, Kir J, Liu D, Bryant D, Guo Y, Stephens R, Baseler MW, Lane HC. DAVID Bioinformatics Resources: expanded annotation database and novel algorithms to better extract biology from large gene lists. Nucleic Acids Res. 2007; 35:W169-W175.

25. Hunter S, Apweiler R, Attwood TK, Bairoch A, Bateman A, Binns D, Bork P, Das U, Daugherty L, Duquenne L. InterPro: the integrative protein signature database. Nucleic Acids Res. 2009; 37:D211-D215.

26. Varley J. Germline TP53 mutations and Li-Fraumeni syndrome. Hum Mutat. 2003; 21:313-320.

27. Bell DA, Taylor JA, Paulson DF, Robertson CN, Mohler JL, Lucier GW. Genetic risk and carcinogen exposure: a common inherited defect of the carcinogen-metabolism gene glutathione S-transferase M1 (GSTM1) that increases susceptibility to bladder cancer. J Natl Cancer Inst. 1993; 85:1159-1164.

28. Bonadona V, Bonaïti B, Olschwang S, Grandjouan S, Huiart L, Longy M, Guimbaud R, Buecher B, Bignon YJ, Caron O. Cancer risks associated with germline mutations in MLH1, MSH2, and MSH6 genes in Lynch syndrome. JAMA. 2011; 305:2304-2310.

29. Chen J, Bardes EE, Aronow BJ, Jegga AG. ToppGene Suite for gene list enrichment analysis and candidate gene prioritization. Nucleic Acids Res. 2009; 37:W305-W311.

30. Zheng S, Zhao Z. GenRev: exploring functional relevance of genes in molecular networks. Genomics. 2012; 99:183-188. 